\title{
Linear and Non-linear Feedback Control Strategies for a 4D Hyperchaotic System
}

\author{
Maysoon M. Aziz, Saad Fawzi AL-Azzawi* \\ Department of Mathematics, College of Computer Sciences and Mathematics, University of Mosul, Mosul, Iraq
}

Email address:

saad_fawzi78@yahoo.com (S. F.AL-Azzawi)

${ }^{*}$ Corresponding author

To cite this article:

Maysoon M. Aziz, Saad Fawzi AL-Azzawi. Linear and Non-linear Feedback Control Strategies for a 4D Hyperchaotic System. Pure and Applied Mathematics Journal. Vol. 6, No. 1, 2017, pp. 5-13. doi: 10.11648/j.pamj.20170601.12

Received: January 1, 2017; Accepted: January 13, 2017; Published: February 16, 2017

\begin{abstract}
This paper investigates the stabilization of unstable equilibrium for a 4D hyperchaotic system. The linear, nonlinear and speed feedback controls are used to suppress hyperchaos to this equilibrium. The Routh-Hurwitz theorem and Lyapunov's second methods are used to derive the conditions of the asymptotic stability of the controlled hyperchaotic system. Theoretical analysis, numerical simulation and illustrative examples are given to demonstrate the effectiveness of the proposed controllers.
\end{abstract}

Keywords: Chaos Control, Linear Feedback Control, Non-linear Feedback Control, Routh-Hurwitz Method, Lyapunov's Second Method

\section{Introduction}

Chaos is an important topic in nonlinear science. In many cases, chaos is sometimes undesirable, so we wish to avoid and eliminate such behaviors [1]. Chaos control and chaos synchronization were once believed to be impossible until the 1990s when Ott et al. developed the OGY method to suppress chaos. Recently, many different techniques and methods have been proposed to achieve chaos control such as impulsive control method, sliding method control, differential geometric method, $H_{\infty}$ control, adaptive control method, and so on. Among them, the feedback control is especially attractive and has been commonly applied to practical implementation due to its simplicity in configuration and implementation [2]. Generally speaking, there are two main approaches for controlling chaos: linear feedback control and non- linear feedback control. The linear feedback control approach offers many advantages such as robustness and computational complexity over the non- feedback control method [2-7].

Many attempts have been made to control hyperchaos of hyperchaotic systems. Recently, Yan (2005)[8], Dou and Sun et al. (2009) [9], Zhu (2010)[10] and Aziz and AL-Azzawi (2015) [11] suppressed hyperchaotic systems to unstable equilibrium by using feedback control method. In 2017, the
Ref. [12] present some problems of these strategies and how treatment. Our system is generated based on Pan system via a state feedback controller which is described by the following mathematical model:

$$
\left\{\begin{array}{c}
\dot{\mathrm{x}}=a(\mathrm{y}-\mathrm{x})+\mathrm{w} \\
\dot{\mathrm{y}}=c \mathrm{x}-\mathrm{xz} \\
\dot{\mathrm{z}}=\mathrm{xy}-b \mathrm{z} \\
\dot{\mathrm{w}}=-d \mathrm{y}
\end{array}\right.
$$

where $(x, y, z, w) \in R^{4}$, and $a, b, c, d \in R$ are constant parameters. When parameters $a=10, b=8 / 3, c=28$ and $d=10$, system (1) is hyperchaotic and has two Lyapunov exponents, i.e. $L E_{\varsigma_{1}}=0.3627, L E_{2}=0.0008$ [13], and hyperchaotic attractors and Lyapunov exponents spectrum are shown in Figure 1 and Figure 2 respectively. The system (1) has only one equilibrium $\mathrm{O}(0,0,0,0)$ and the equilibrium is an unstable under these parameters [13].

The aim of this paper is discuss three feedback control methods for a 4D hyperchaotic system. The rest of the paper is organized as follows. section 2 present helping results which inclined ordinary feedback control, dislocated feedback control, enhancing feedback control, and speed feedback control. main results are present in section 3. And section 4 present the flow chats for linear and non-linear feedback control. Finally, the conclusion are given in section 
5. Moreover, numerical simulations and illustrative examples are applied to verify the effectiveness of chosen controllers.

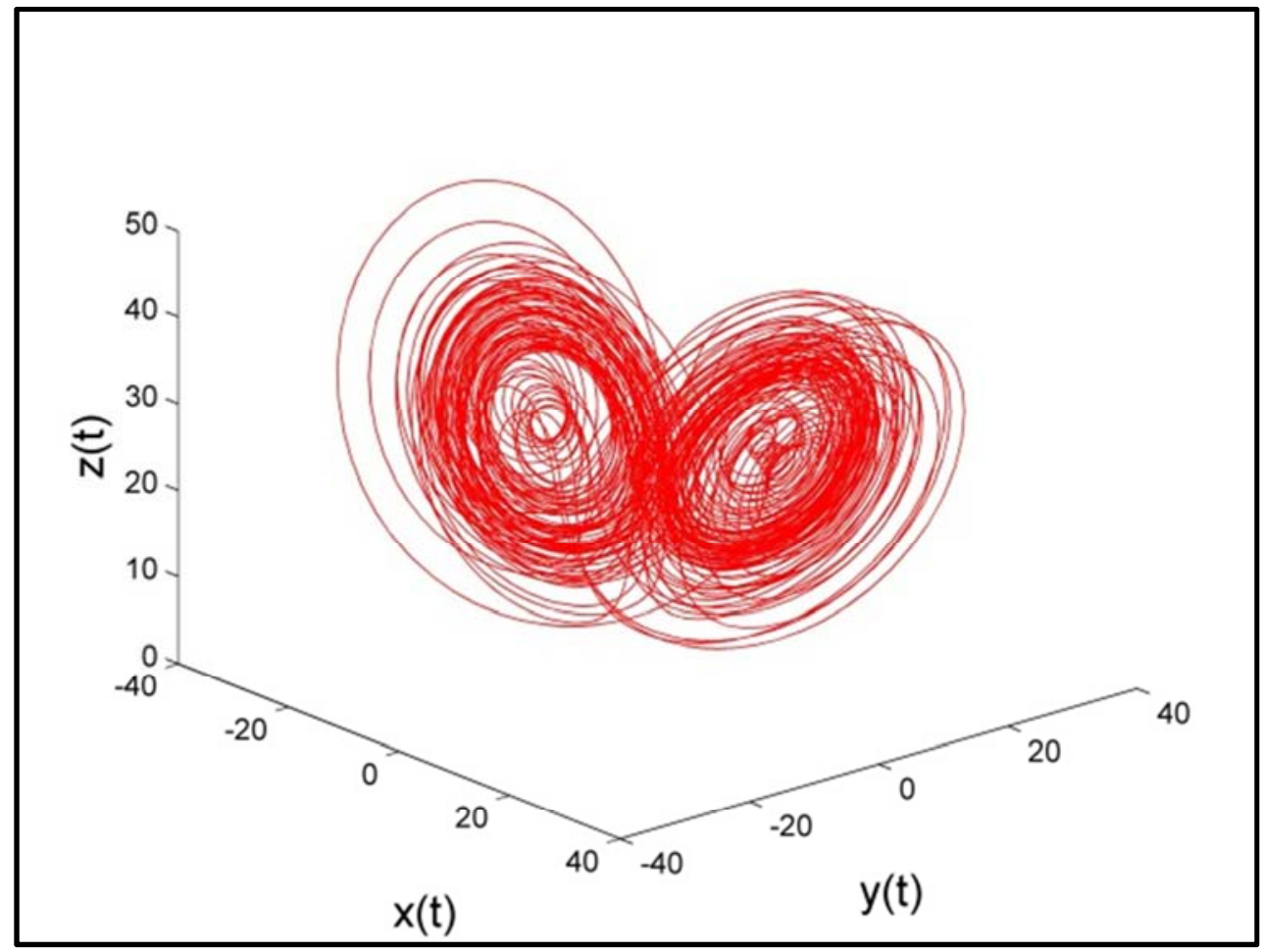

Figure 1. The attractor of system (1) in $x-y-z$ space.

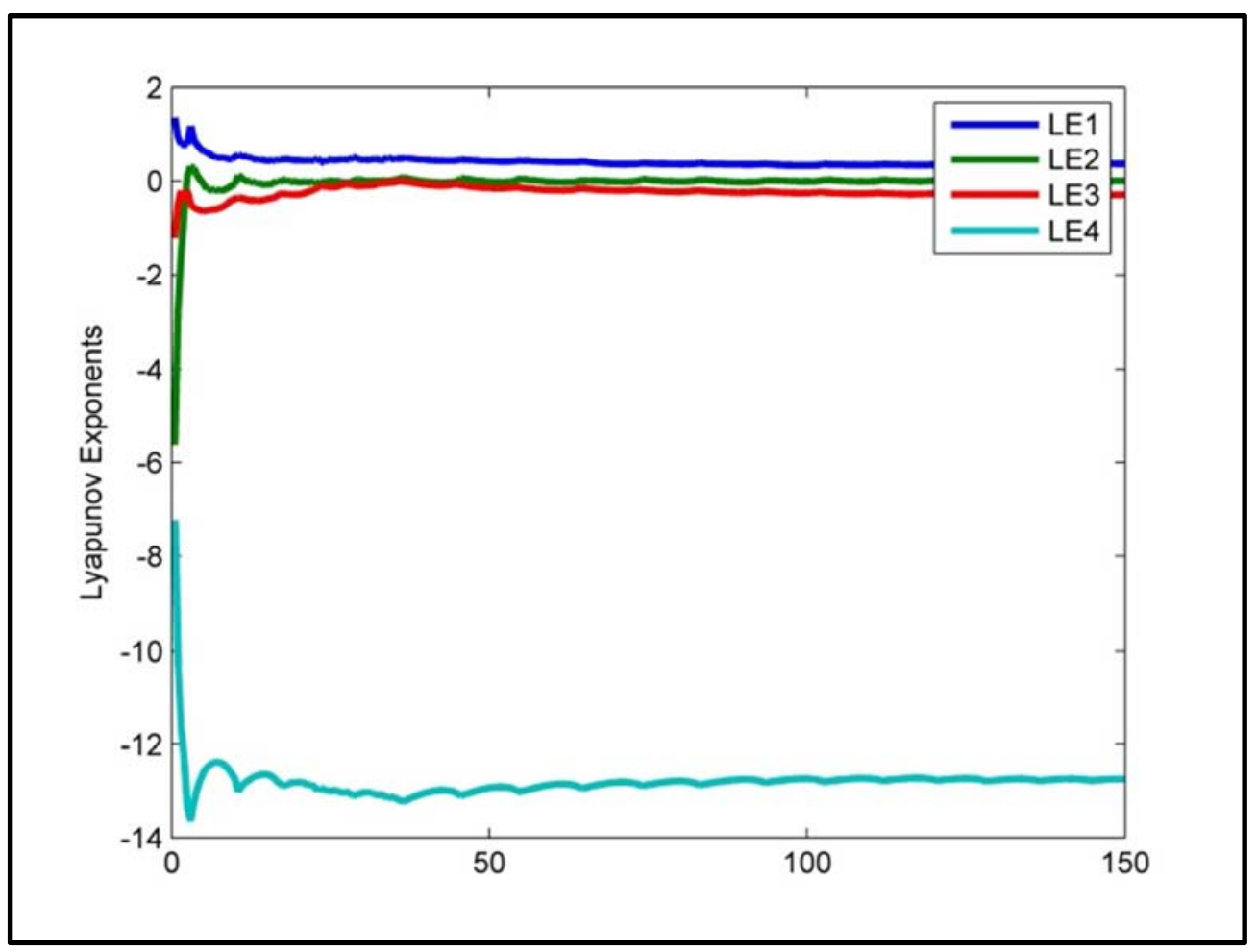

Figure 2. The Lyapunov exponents of system (1).

\section{Helping Results}

To control the hyperchaotic system (1) to the unstable equilibrium $\mathrm{O}(0,0,0,0)$, we use the feedback control approach to control it. Let us assume that the controlled hyperchaotic system (1) is given by

$$
\left\{\begin{array}{c}
\dot{\mathrm{x}}=10(\mathrm{y}-\mathrm{x})+\mathrm{w}+\mathrm{u}_{1} \\
\dot{\mathrm{y}}=28 \mathrm{x}-\mathrm{xz}+\mathrm{u}_{2} \\
\dot{\mathrm{z}}=\mathrm{xy}-8 / 3 \mathrm{z}+\mathrm{u}_{3} \\
\dot{\mathrm{w}}=-10 \mathrm{y}+\mathrm{u}_{4}
\end{array}\right.
$$


where $\mathrm{u}_{1}, \mathrm{u}_{2}, \mathrm{u}_{3}$ and $\mathrm{u}_{4}$ are external feedback control inputs which will be suitably derive the trajectory of the hyperchaotic system, specified by $(x, y, z, w)$ to the equilibrium $\mathrm{O}(0,0,0,0)$ of uncontrolled system (i.e. $\mathrm{u}_{i}=$ $0, \mathrm{i}=1,2,3,4)$.

Remark 1 (Ordinary feedback control method)

For the ordinary feedback control, the system variable is often multiplied by a coefficient as the feedback gain, and the feedback gain is added to the right -hand of the corresponding equation [3-5, 7, 10-12, 16].

Remark 2 (Dislocated feedback control method)

If a system variable multiplied by a coefficient is added to the right -hand of another equation, then this method is called dislocated feedback control [1, 3-5, 7, 10-12, 16].

Remark 3 (Enhancing feedback control method)

It is difficult for a complex system to be controlled by only one feedback variable, and in such cases the feedback gain is always very large. So we consider using multiple variables multiplied by a proper coefficient as the feedback gain. This method is called enhancing feedback control $[1,3-5,7,10-$ $12,16]$.

\section{Remark 4.}

The above three remarks are used in linear feedback control, also can use with nonlinear feedback control, but in nonlinear feedback the system variable multiplied by a quantity which contains coefficient and nonlinear term and added to the right -hand of equation according to each above three remarks.

Remark 5 (Speed feedback control method)

For the feedback control, the independent variable of a system function is often multiplied by a coefficient as the feedback gain, so the method is called displacement feedback control. Similarly, if the derivative of an independent variable is multiplied by a coefficient as the feedback gain, it is called speed feedback control [2-12, 16].

Remark 6 (Routh-Hurwitz method, [14]). All the roots of the indicated polynomial have negative real parts precisely when the given conditions are met.

- $\lambda^{2}+A \lambda+\mathrm{B}: \mathrm{A}>0, \mathrm{~B}>0$

- $\lambda^{3}+A \lambda^{2}+B \lambda+\mathrm{C}: \mathrm{A}>0, \mathrm{C}>0, \mathrm{AB}-\mathrm{C}>0$

- $\lambda^{4}+\mathrm{A} \lambda^{3}+B \lambda^{2}+C \lambda+\mathrm{D}: \mathrm{A}>0, \mathrm{AB}-\mathrm{C}>$ $0,(\mathrm{AB}-\mathrm{C}) \mathrm{C}-\mathrm{A}^{2} \mathrm{D}>0, \mathrm{D}>0$

Remark 7 (Barbalat Remark, [15, 16]).

If $f(t) \epsilon L_{2} \cup L_{\infty}$ and $\dot{f}(t) \epsilon L_{\infty}$, then $\lim _{t \rightarrow \infty} f(t)=0$.

\section{Main Results}

Theorem 1 (Ordinary feedback control method).

Let the controlled hyperchaotic system (2) be

$$
\left\{\begin{array}{c}
\dot{\mathrm{x}}=a(\mathrm{y}-\mathrm{x})+\mathrm{w} \\
\dot{\mathrm{y}}=c \mathrm{x}-\mathrm{xz}-\mathbf{k y} \\
\dot{\mathrm{z}}=\mathrm{xy}-b \mathrm{z} \\
\dot{\mathrm{w}}=-d \mathrm{y}
\end{array}\right.
$$

where $k$ is the feedback coefficient. When

$$
\mathrm{k}>\frac{-\mathrm{a}(\mathrm{a}-\mathrm{c})+\sqrt{\mathrm{a}\left(\mathrm{a}^{3}+\mathrm{ac}^{2}+2 \mathrm{a}^{2} \mathrm{c}+4 \mathrm{~cd}\right)}}{2 \mathrm{a}}, \text { system (3) will gradually }
$$

converge to the unstable equilibrium $\mathrm{O}(0,0,0,0)$.

Proof. The Jacobi matrix of system (3) is

$$
J=\left[\begin{array}{cccc}
-a & a & 0 & 1 \\
\mathrm{c} & -\mathrm{k} & 0 & 0 \\
0 & 0 & -b & 0 \\
0 & -\mathrm{d} & 0 & 0
\end{array}\right]
$$

The characteristic equation of matrix (4) is

$$
(\lambda+\mathrm{b})\left(\lambda^{3}+A \lambda^{2}+B \lambda+\mathrm{C}\right)=0,
$$

where $A=k+a, B=a k-a c, C=c d$

According to the Routh-Hurwitz method, the Jacobian matrix of system (4) has four negative real part eigenvalues if and only if $k+a>0, c d>0$,

$$
(k+a)(a k-a c)-c d>0
$$

Implying $A, C>0$ since $a, c$ and $d>0$ (given) and $k$ is always positive and $A B-C>0$ it is possible under the condition $\mathrm{k}>\frac{-\mathrm{a}(\mathrm{a}-\mathrm{c})+\sqrt{\mathrm{a}\left(\mathrm{a}^{3}+\mathrm{ac}^{2}+2 \mathrm{a}^{2} \mathrm{c}+4 \mathrm{~cd}\right)}}{2 \mathrm{a}}$.

Thus system (3) will gradually converge to the unstable equilibrium $\mathrm{O}(0,0,0,0)$. The proof is completed.

Illustrative Example 1. the hyperchaotic system (3) be

$$
\left\{\begin{array}{c}
\dot{\mathrm{x}}=10(\mathrm{y}-\mathrm{x})+\mathrm{w} \\
\dot{\mathrm{y}}=28 \mathrm{x}-\mathrm{xz}-\boldsymbol{k y} \\
\dot{\mathrm{z}}=\mathrm{xy}-8 / 3 \mathrm{z} \\
\dot{\mathrm{w}}=-10 \mathrm{y}
\end{array}\right.
$$

When $k>28.7231$, system (6) will gradually converge to the unstable equilibrium $\mathrm{O}(0,0,0,0)$ according to theorem 1 .

Sol. The characteristic equation of system (6) is

$$
(\lambda+8 / 3)\left(\lambda^{3}+(10+k) \lambda^{2}+(10 k-280) \lambda+280\right)=0,
$$

Then the roots of equation (7) depended of the value of $k$ as following:

when $k=29$, the equation (7) became

$$
(\lambda+8 / 3)\left(\lambda^{3}+39 \lambda^{2}+10 \lambda+280\right)=0,
$$

and the all roots have negative real parts

$$
\lambda_{1}=-\frac{8}{3}, \lambda_{2}=-38.9279, \lambda_{3,4}=-0.0361 \pm 2.6817 i,
$$
therefore the system (6) is asymptotically stable.

while $k=28.5$, the equation (7) became

$$
(\lambda+8 / 3)\left(\lambda^{3}+38.5 \lambda^{2}+5 \lambda+280\right)=0,
$$

then not all roots have negative real parts

$$
\lambda_{1}=-\frac{8}{3}, \lambda_{2}=-38.5587, \lambda_{3,4}=0.0293 \pm 2.6946 i
$$

therefore the system (6) is unstable.

Also can justified the same result that obtain in theorem 1 by using MATLAB software where numerical simulations are used to investigate the controlled hyperchaotic system (3) using fourth-order Runge-Kutta scheme with time step 0.1 . The initial values are taken as by $[x(0)=-5, y(0)=$ $5, z(0)=10, w(0)=15]$. The feedback coefficients are given by $k=29, k=28.5$ in Figure $3-\mathrm{a}$, b respectively. The behaviors of the states $(x(t), y(t), z(t), w(t))$ of the control 
hyperchaotic system (6) show converging to $0(0,0,0,0)$ when $\quad k=28.5$ (Figure $3, \mathrm{~b}$.) $k=29$ (Figure 3,a.) and divergence to $0(0,0,0,0)$ when

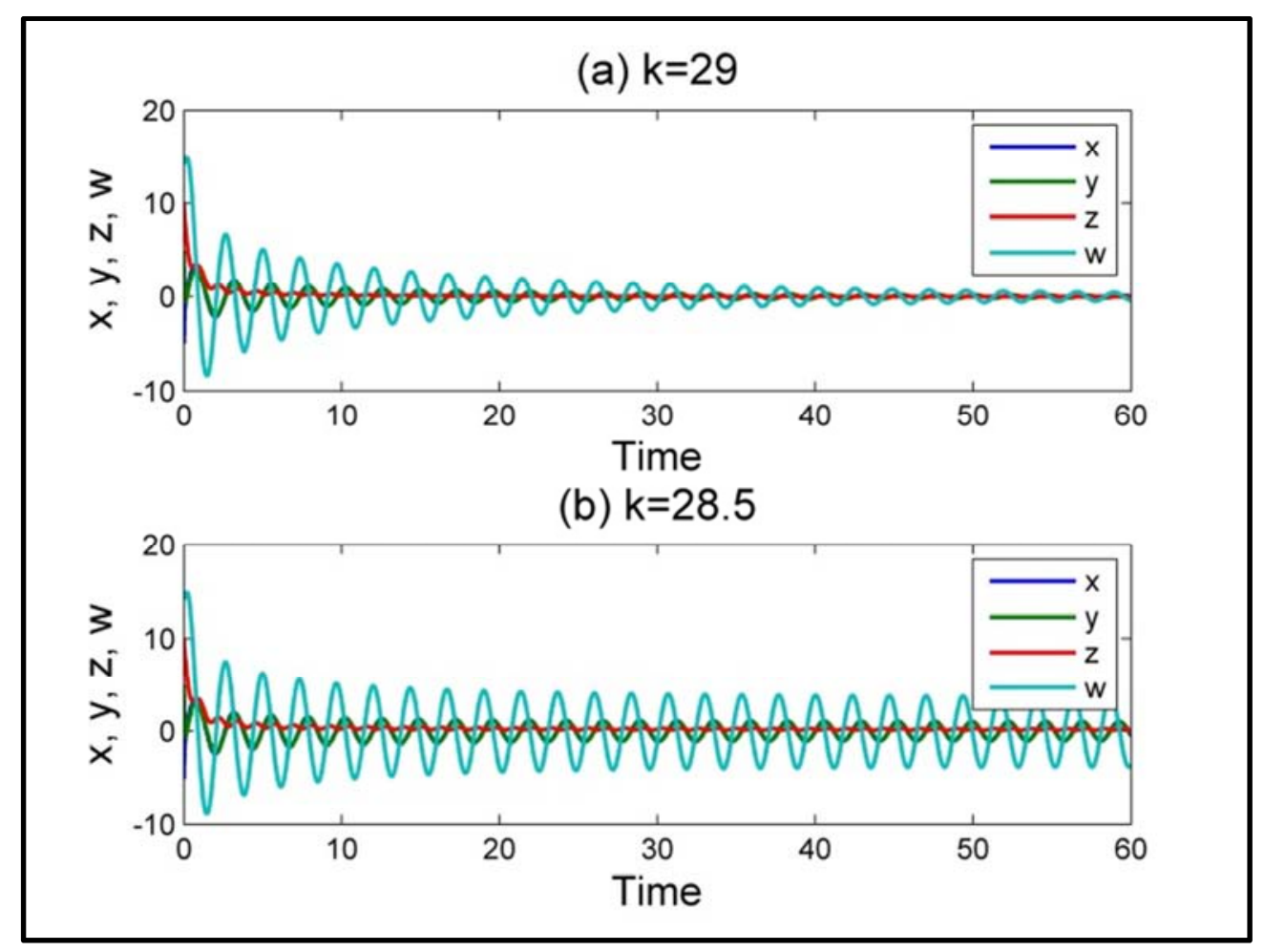

Figure 3. The difference of the state of the controlled system (3) with the control gain change.

Theorem 2 (Dislocated feedback control method).

Let $\mathrm{u}_{1}=-k y, \mathrm{u}_{2}=\mathrm{u}_{3}=\mathrm{u}_{4}=0$ and the controlled hyperchaotic system (2) be

$$
\left\{\begin{array}{c}
\dot{\mathrm{x}}=a(\mathrm{y}-\mathrm{x})+\mathrm{w}-\mathbf{k y} \\
\dot{\mathrm{y}}=c \mathrm{x}-\mathrm{xz} \\
\dot{\mathrm{z}}=\mathrm{xy}-b \mathrm{z} \\
\dot{\mathrm{w}}=-d \mathrm{y}
\end{array}\right.
$$

where $k$ is the feedback coefficient. When $k>\frac{a^{2}+d}{a}$, system (8) will gradually converge to the unstable equilibrium $\mathrm{O}(0,0,0,0)$.

Proof. The Jacobi matrix of system (8) is

$$
J=\left[\begin{array}{cccc}
-a & a-k & 0 & 1 \\
\mathrm{c} & 0 & 0 & 0 \\
0 & 0 & -b & 0 \\
0 & -\mathrm{d} & 0 & 0
\end{array}\right]
$$

The characteristic equation of matrix (9) is

$$
(\lambda+\mathrm{b})\left(\lambda^{3}+A \lambda^{2}+B \lambda+\mathrm{C}\right)=0,
$$

where $A=a, B=c k-a c, C=c d$

By using Routh-Hurwitz method, the Jacobian matrix of system (9) has four negative real part eigenvalues if and only if $a>0, c d>0 a(c k-a c)-c d>0$,

Implying $A, C>0$ since a, c and $\mathrm{d}$ are positive (given) and $\mathrm{k}$ is always positive and $A B-C>0$, it is possible under the condition $k>\frac{a^{2}+d}{a}$. Thus system (8) will gradually converge to the unstable equilibrium $\mathrm{O}(0,0,0,0)$. The proof is completed.

Illustrative Example 2: Let the hyperchaotic system (8) be

$$
\left\{\begin{array}{c}
\dot{x}=10(y-x)+w-k y \\
\dot{y}=28 x-x z \\
\dot{z}=x y-8 / 3 z \\
\dot{w}=-10 y
\end{array}\right.
$$

When $k>11$, system (11) will gradually converge to the unstable equilibrium $\mathrm{O}(0,0,0,0)$ according to theorem 2 .

Sol. The characteristic equation of system (11) is

$$
(\lambda+8 / 3)\left(\lambda^{3}+10 \lambda^{2}+(28 k-280) \lambda+280\right)=0
$$

Then the roots of equation (12) depended of the value of $k$ as following:

when $k=11.1$, the equation (12) became

$$
(\lambda+8 / 3)\left(\lambda^{3}+10 \lambda^{2}+30.8 \lambda+280\right)=0,
$$

and the all roots have negative real parts

$$
\lambda_{1}=-\frac{8}{3}, \lambda_{2}=-9.7785, \lambda_{3,4}=-0.01107 \pm 5.3499 i
$$
therefore the system (11) is asymptotically stable.

While $k=10.9$, the equation (12) became

$$
(\lambda+8 / 3)\left(\lambda^{3}+10 \lambda^{2}+25.2 \lambda+280\right)=0,
$$

then not all roots have negative real parts

$$
\lambda_{1}=-\frac{8}{3}, \lambda_{2}=-10.2161, \lambda_{3,4}=0.1081 \pm 5.2341 i
$$

therefore the system (11) is unstable. 
The feedback coefficients are given by $k=11.1, k=10.9$ in Figure 4- a, b respectively. The behaviors of the states $(x(t), y(t), z(t), w(t))$ of the control hyperchaotic system
(11) show converging to $\mathrm{O}(0,0,0,0)$. when $k=11.1$ (Figure 4, a.) and divergence to $0(0,0,0,0)$ when $k=10.9$ in (Figure 4,b.).

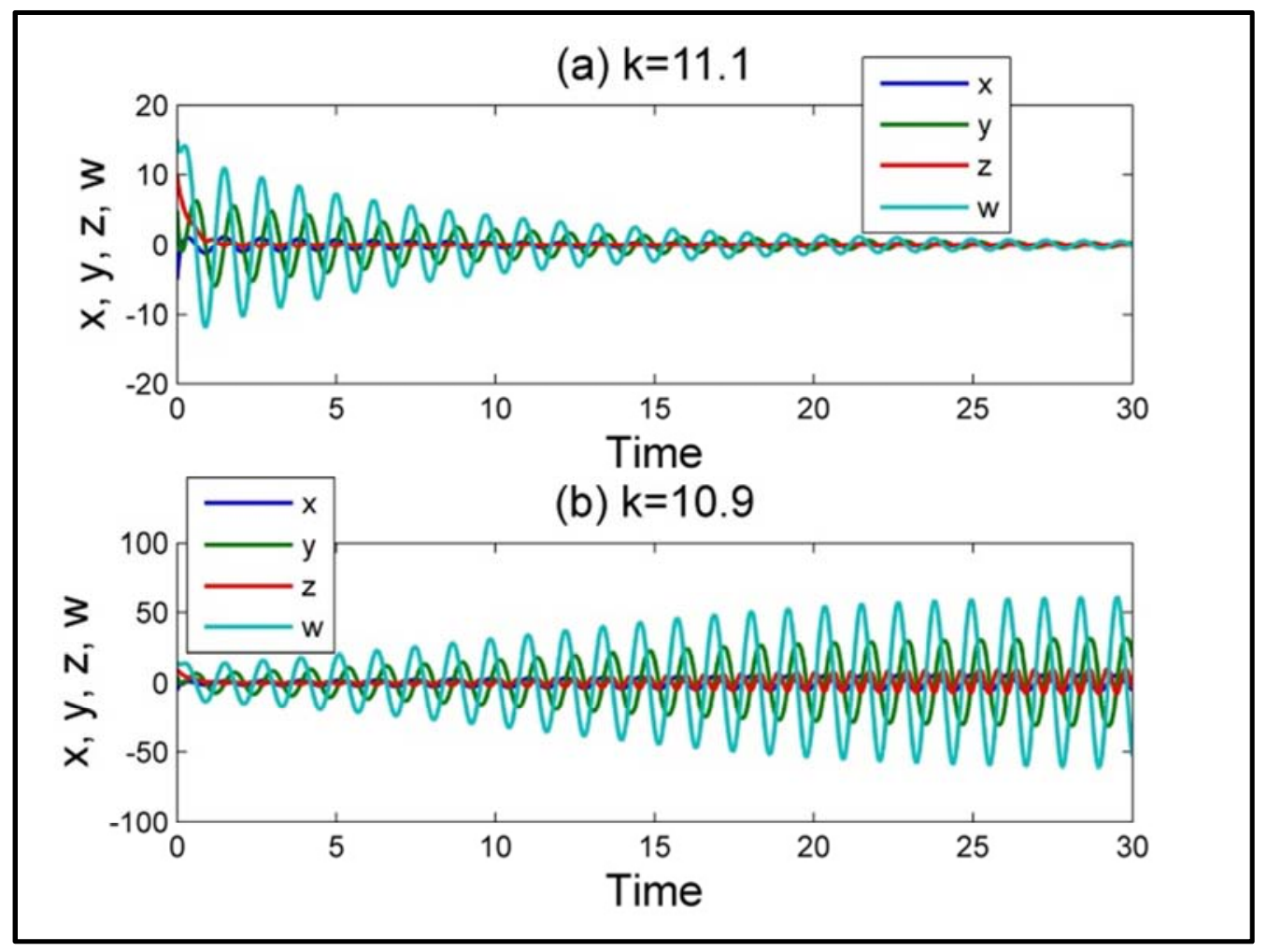

Figure 4. The difference of the state of the controlled system (11) with the control gain change.

Theorem 3 (Enhancing feedback control method):

Let $\mathrm{u}_{1}=-k x, \mathrm{u}_{2}=-k y, \mathrm{u}_{3}=\mathrm{u}_{4}=0$ and the controlled hyperchaotic system (2) be

$$
\left\{\begin{array}{c}
\dot{\mathrm{x}}=a(\mathrm{y}-\mathrm{x})+\mathrm{w}-\mathbf{k x} \\
\dot{\mathrm{y}}=c \mathrm{x}-\mathrm{xz}-\mathbf{k y} \\
\dot{\mathrm{z}}=\mathrm{xy}-b \mathrm{z} \\
\dot{\mathrm{w}}=-d \mathrm{y}
\end{array}\right.
$$

where $k$ is the feedback coefficient.

When $2 k^{3}+3 a k^{2}+\left(a^{2}-2 a c\right) k-c\left(a^{2}+d\right)>0$, system (13) will gradually converge to the unstable equilibrium $\mathrm{O}(0,0,0,0)$.

Proof. Analogously as in proof of Theorem 1 and Theorem
Illustrative Example 3: Let the hyperchaotic system (13) be

$$
\left\{\begin{array}{c}
\dot{x}=10(y-x)+w-\mathbf{k x} \\
\dot{y}=28 x-x z-\mathbf{k y} \\
\dot{z}=x y-\left(\frac{8}{3}\right) z \\
\dot{w}=-10 y
\end{array}\right.
$$

When $k>12.6894$, system (14) will gradually converge to the unstable equilibrium $\mathrm{O}(0,0,0,0)$ according to theorem 3 .

Sol. The characteristic equation of system (14) is 2.

$$
(\lambda+8 / 3)\left(\lambda^{3}+(2 k+10) \lambda^{2}+\left(k^{2}+10 \mathrm{k}-280\right) \lambda+280\right)=0,
$$

Then the roots of equation (15) depended of the value of $\mathrm{k}$ as following:

when $k=13$, the equation (15) became

$$
(\lambda+8 / 3)\left(\lambda^{3}+36 \lambda^{2}+19 \lambda+280\right)=0,
$$

and the all roots have negative real parts

$$
\lambda_{1}=-\frac{8}{3}, \lambda_{2}=-35.6875, \lambda_{3,4}=-0.1563 \pm 2.7967 i
$$

therefore the system (14) is asymptotically stable.

while $k=12$, the equation (15) became

$$
(\lambda+8 / 3)\left(\lambda^{3}+34 \lambda^{2}-16 \lambda+280\right)=0,
$$

then not all roots have negative real parts

$$
\lambda_{1}=-\frac{8}{3}, \lambda_{2}=-34.6938, \lambda_{3,4}=0.3469 \pm 2.8196 i
$$

therefore the system (14) is unstable.

The feedback coefficients are given by $k=13, k=12$ in Figure 5- a, b respectively. The behaviors of the states $(x(t), y(t), z(t), w(t))$ of the control hyperchaotic system (14) show converging to $0(0,0,0,0)$ when $k=13$ (Figure 5 ,a.) and divergence to $\mathrm{O}(0,0,0,0)$ when $k=12$ (Figure 5,b.). 


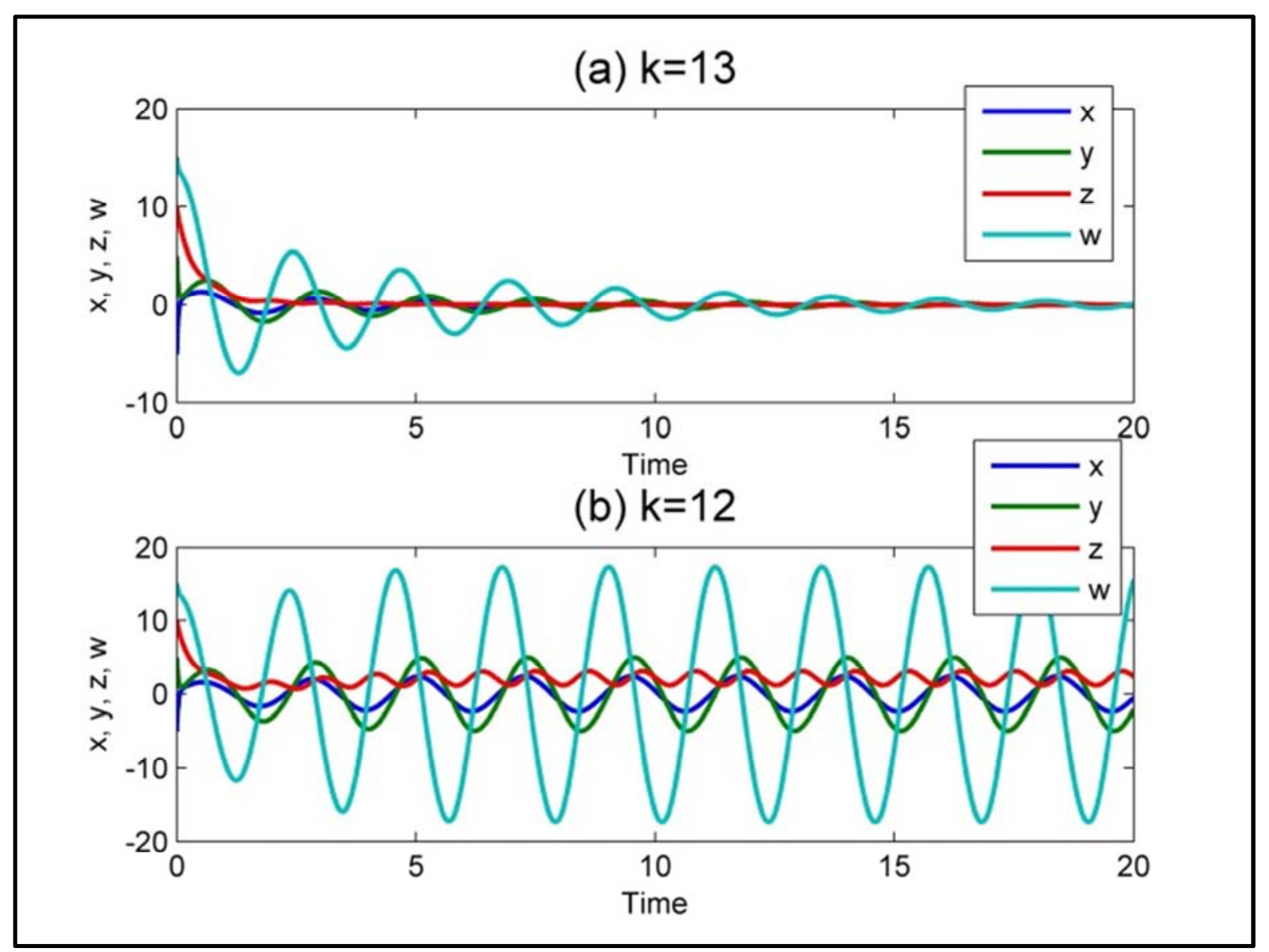

Figure 5. The difference of the state of the controlled system (14) with the control gain change.

Theorem 4 (Speed feedback control method).

Let $\mathrm{u}_{4}=-k \dot{y}, \mathrm{u}_{1}=\mathrm{u}_{2}=\mathrm{u}_{3}=0$ and the controlled hyperchaotic system be

$$
\left\{\begin{array}{c}
\dot{\mathrm{x}}=a(\mathrm{y}-\mathrm{x})+\mathrm{w} \\
\dot{\mathrm{y}}=c \mathrm{x}-\mathrm{xz} \\
\dot{\mathrm{z}}=\mathrm{xy}-b \mathrm{z} \\
\dot{\mathrm{w}}=-d \mathrm{y}-\mathrm{ky}
\end{array}\right.
$$

where $k$ is the feedback coefficient. When $k>\frac{a^{2}+d}{a}$, system (16) will gradually converge to the unstable equilibrium $\mathrm{O}(0,0,0,0)$. 2 .

Proof. Analogously as in proof of Theorem 1 and Theorem

Illustrative Example 4. Let the hyperchaotic system (16) be

$$
\left\{\begin{array}{c}
\dot{x}=10(y-x)+w \\
\dot{y}=28 x-x z \\
\dot{z}=x y-\left(\frac{8}{3}\right) z \\
\dot{w}=-10 y-k \dot{y}
\end{array}\right.
$$

When $k>11$, system (17) will gradually converge to the unstable equilibrium $\mathrm{O}(0,0,0,0)$ according to theorem 4 .

Sol. The characteristic equation of system (17) is

$$
(\lambda+8 / 3)\left(\lambda^{3}+10 \lambda^{2}+(28 k-280) \lambda+280\right)=0
$$

Then the roots of equation (18) depended of the value of $k$ as following:

when $k=11.5$, the equation (18) became

$$
(\lambda+8 / 3)\left(\lambda^{3}+10 \lambda^{2}+42 \lambda+280\right)=0,
$$

and the all roots have negative real parts

$$
\lambda_{1}=-\frac{8}{3}, \lambda_{2}=-8.8337, \lambda_{3,4}=-0.5832 \pm 5.5997 i
$$

therefore the system (17) is asymptotically stable. while $k=10.5$, the equation (18) became

$$
(\lambda+8 / 3)\left(\lambda^{3}+10 \lambda^{2}+14 \lambda+280\right)=0,
$$

then not all roots have negative real parts

$$
\lambda_{1}=-\frac{8}{3}, \lambda_{2}=-11.0317, \lambda_{3,4}=0.5158 \pm 5.0115 i
$$

therefore the system (17) is unstable.

The behaviors of the states $(x(t), y(t), z(t), w(t))$ of the control hyperchaotic system (17) show converging to $\mathrm{O}(0,0,0,0)$ when $k=11.5$ (Figure 6,a) and divergence to $\mathrm{O}(0,0,0,0)$ when $k=10.5$ (Figure 6,b). 


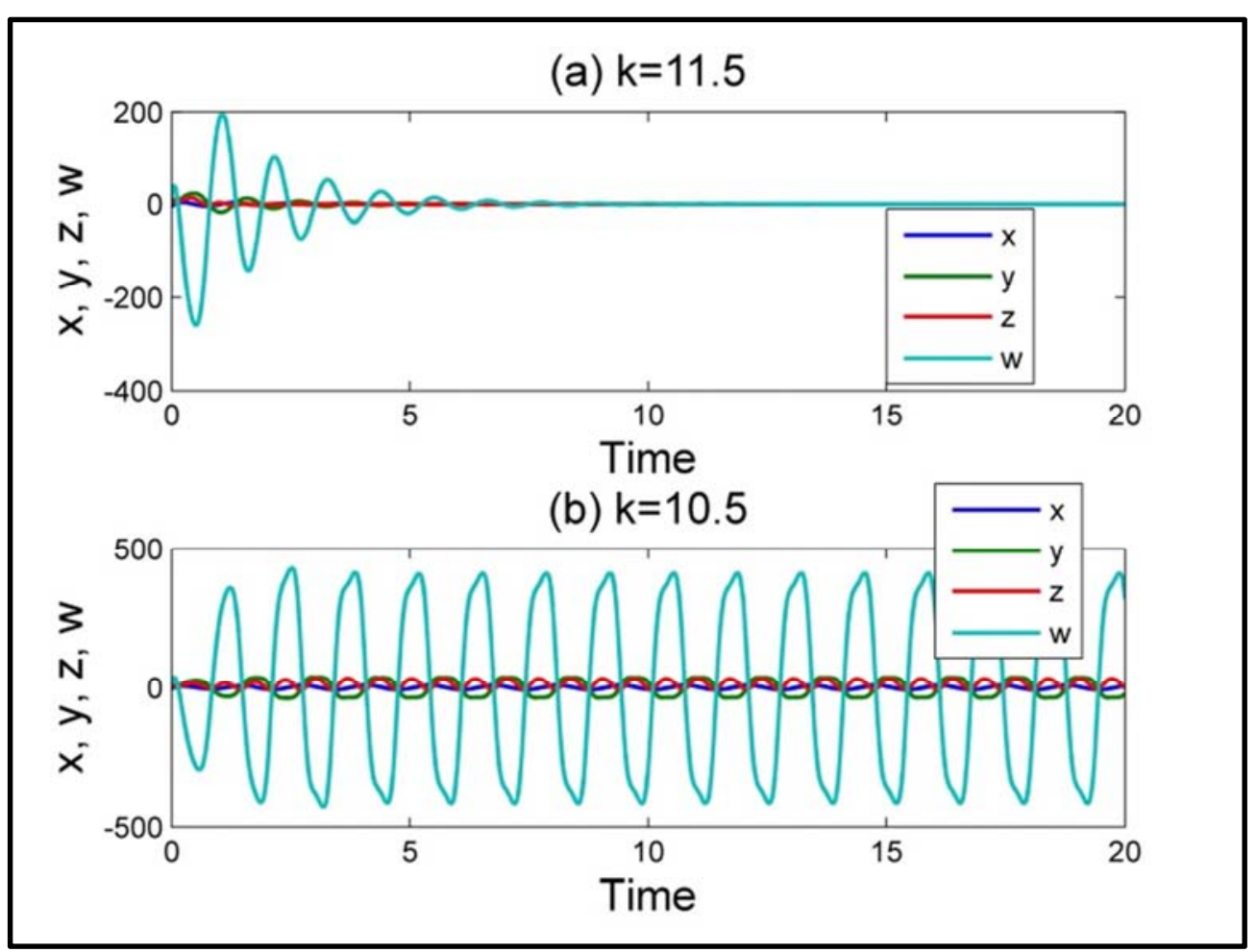

Figure 6. The difference of the state of the controlled system (17) with the control gain change.

Theorem 5 (Nonlinear feedback control method).

Consider the controlled hyperchaotic system (2) as follows:

$$
\left\{\begin{array}{c}
\dot{\mathrm{x}}=a(\mathrm{y}-\mathrm{x})+\mathrm{w} \\
\dot{\mathrm{y}}=c \mathrm{x}-\mathrm{xz}-\hat{k}_{1} y \\
\dot{\mathrm{z}}=\mathrm{xy}-b \mathrm{z} \\
\dot{\mathrm{w}}=-d \mathrm{y}-\hat{k}_{2} w
\end{array}\right.
$$

Where $\dot{\hat{k}}_{1}=\beta_{1} y^{2}, \dot{\hat{k}}_{2}=\beta_{2} w^{2}, \beta_{1}, \beta_{2}$ are a positive feedback gain, then system (19) will converge to the unstable equilibrium point $\mathrm{O}(0,0,0,0)$.

Proof. Construct the Lyapunov function

$$
\begin{gathered}
V=\frac{1}{2}\left(x^{2}+y^{2}+z^{2}+w^{2}\right)+\frac{1}{2 \beta_{1}}\left(\hat{k}_{1}-k^{*}\right)^{2}+\frac{1}{2 \beta_{2}}\left(\hat{k}_{2}\right. \\
\left.-k^{*}\right)^{2}
\end{gathered}
$$

where $k^{*}$ is a parameter to be determined.

The derivation of $V$ is:

$$
\begin{aligned}
\dot{V}=x \dot{x}+y \dot{y}+z \dot{z}+w \dot{w}+\left(\hat{k}_{1}-k^{*}\right) y^{2}+\left(\hat{k}_{2}-k^{*}\right) w^{2} \\
k^{*}>\max \left\{\frac{(a+c)^{2}}{4 a}, \frac{1+(a+c)^{2}+\sqrt{\left(1+(a+c)^{2}\right)^{2}-16 a\left((a+c) d-a d^{2}\right.}}{8 a}\right\}
\end{aligned}
$$

so that $V$ is positive definite and $\dot{V}$ is negative semi-definite. However, cannot get immediately the controlled system (19) is asymptotically stable at equilibrium $\mathrm{O}(0,0,0,0)$ i.e. , $\mathrm{x}, \mathrm{y}, \mathrm{z}, \mathrm{w} \in L_{\infty}$ and $\hat{k}_{1}-k^{*}, \hat{k}_{2}-k^{*} \in L_{\infty}$, for this reason, $\dot{x}, \dot{y}, \dot{z}, \dot{w} \in L_{\infty}$ then, we have

$$
=-a x^{2}-k^{*} y^{2}-b z^{2}-k^{*} w^{2}+(a+c) x y+x w-d y w
$$

$$
=-[x, y, z, w]\left[\begin{array}{cccc}
a & -\frac{a+c}{2} & 0 & -\frac{1}{2} \\
-\frac{a+c}{2} & k^{*} & 0 & \frac{\mathrm{d}}{2} \\
0 & 0 & b & 0 \\
-\frac{1}{2} & \frac{\mathrm{d}}{2} & 0 & k^{*}
\end{array}\right]\left[\begin{array}{c}
x \\
y \\
z \\
w^{2}
\end{array}\right]
$$

To ensure the system (19) asymptotically stable, the symmetric matrix $\mathrm{P}$ should be positive definite, and when $\mathrm{P}$ satisfy the following conditions:

$$
\left\{\begin{array}{c}
\text { 1. } a>0 \\
2 \cdot a k^{*}-\frac{(a+c)}{4}^{2}>0 \\
3 \cdot a b k^{*}-b \frac{(a+c)}{4}^{2}>0 \\
4 \cdot a k^{* 2}-\frac{1}{4}\left(1+(a+c)^{2} k^{*}+\frac{1}{4}\left((a+c) d-a d^{2}\right)>0\right.
\end{array}\right.
$$
we can choose the constant $k^{*}$ as

$$
\begin{aligned}
& \int_{0}^{t} \lambda_{\min }(p)\|x\|^{2} d t \leq \int_{0}^{t} X^{T} P X=\int_{0}^{t}(-\dot{V}) d t=V(0)-v(t) \\
& \leq V(0)
\end{aligned}
$$


where $\lambda_{\min }(p)$ is the smallest eigenvalue of the positive matrix $\mathrm{P}$. and so as $\mathrm{x}, \mathrm{y}, \mathrm{z}, \mathrm{w} \in L_{2}$, according to the Barbalat Lemma, implies $x, y, z, w \rightarrow 0$ as $t \rightarrow 0$, therefore the controlled hyperchaotic system (19) is asymptotically stable to equilibrium point, This completes the proof.

\section{Flow Chart of Linear and Non-linear Feedback Control Strategies}

The following flow charts explains briefly the Linear and non-linear feedback control strategies respectively

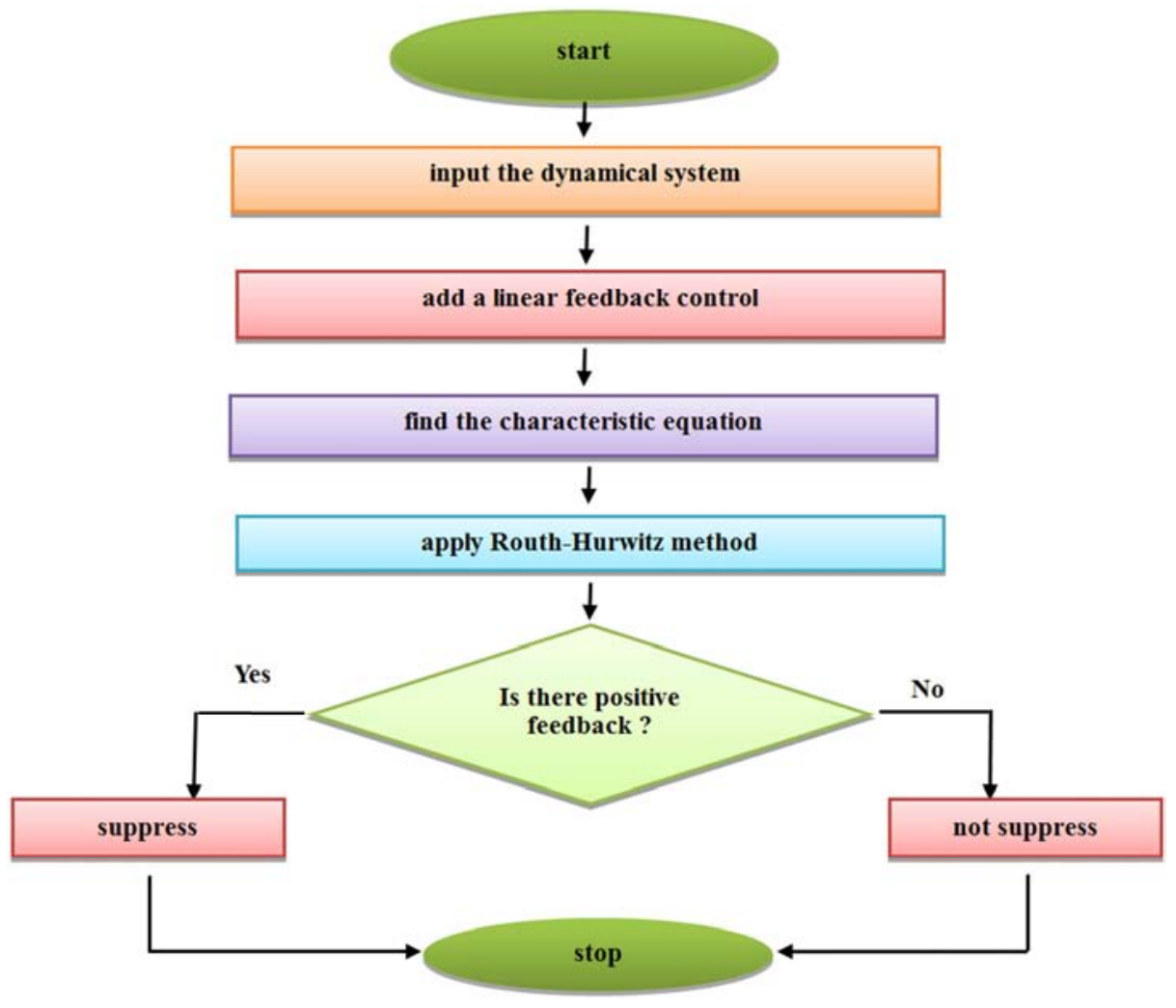

Figure 7. Flow chart of Linear feedback control strategies.

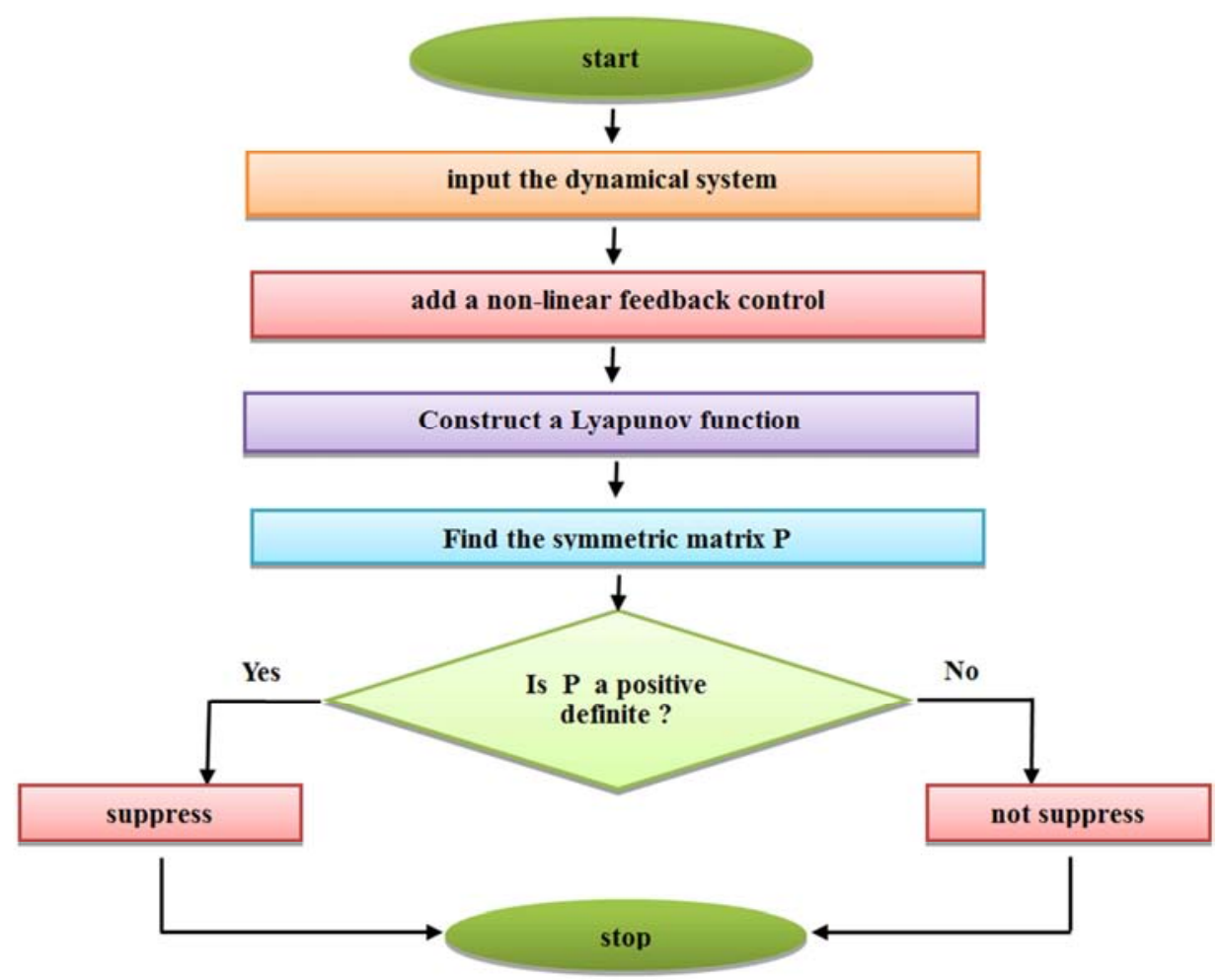

Figure 8. Flow chart of non-Linear feedback control strategies. 


\section{Conclusions}

In this paper, the control problem of a 4D hyperchaotic system is investigated: ordinary feedback control, dislocated feedback control, enhancing feedback control, speed feedback control are used to suppress hyperchaos to unstable equilibrium. The Routh-Hurwitz and Lyapunov's second methods are applied to derive the conditions of the asymptotic stability of the controlled hyperchaotic system. Theoretical analysis,numerical simulation and illustrative examples are given to demonstrate the effectiveness of the proposed controllers.

\section{References}

[1] X. Changjin, L. Peiluan, Chaos control for a 4D hyperchaotic system, Applied Mechanics and Materials. 418 (2013) 84-87.

[2] H. Wang, G. Cai, Controlling hyperchaos in a novel hyperchaotic system, Journal of Information and Computing Science, 4 (2009) 251-258.

[3] C. Yang, C. H. Tao, P. Wang, Comparison of feedback control methods for a hyperchaotic Lorenz system, Physics Letters A 374 (2010) 729-732.

[4] C. Zhu, Feedback control method for stabilizing unstable equilibrium points in a new chaotic system, Nonlinear analysis, 71 (2009) 2441-2446.

[5] C. Zhu, Z. Chen, Feedback control strategies for the Liu chaotic system, Phys. Lett. A 372 (2008) 4033-4036.

[6] C. Tao, C. Yang, Y. Luo, H. Xiong, F. Hu, Speed feedback control of chaotic system, Chaos Solitons Fractals 23 (2005) 259-263.
[7] S. Pang, Y. Liu, A new hyperchaotic system from the L $\ddot{u}$ system and its control. Journal of Computational and Applied Mathematics, 235 (2011) 2775-2789.

[8] Z. Yan, Controlling hyperchaos in the new hyperchaotic chen system, Appl. Math. Comput. 168 (2005) 1239-1250.

[9] Q. Dou, J. A. Sun, W. S. Duan, K. P. Lü, Controlling hyperchaos in the new hyperchaotic system, Commun. Nonlinear Sci. Numer., 14 (2009) 552-559.

[10] C. Zhu, Controlling hyperchaos in hyperchaotic Lorenz system using feedback controllers, Appl. Math. Comput. 216 (2010) 3126-3132.

[11] M. M. Aziz, S. F. AL-Azzawi.. Using Feedback Control Methods to Suppress a Modified Hyperchaotic Pan System, Computational and Applied Mathematics Journal. 1 (3), (2015) 97-106.

[12] M. M. Aziz, S. F. AL-Azzawi, Some Problems of feedback control strategies and its treatment, Journal of Mathematics Research; 9 (1), (2017) 39-49.

[13] S. F. AL-Azzawi, Study of dynamical properties and effective of a state $\mathrm{u}$ for hyperchaotic pan systems, Al-Rafiden J. Comput. Sci. Math. 10 (2013) 89-99.

[14] S. F. AL-Azzawi, Stability and bifurcation of pan chaotic system by using Routh-Hurwitz and Gardan method, Appl. Math. Comput. 219 (2012) 1144-1152.

[15] W. Xiang, C. Liang, M. Sheng, T. Xin, Nonlinear feedback control of a novel hyperchaotic system and its circuit implementation, 19 (2010)

[16] C. Tao, C. Yang, Three control strategies for the Lorenz chaotic system, Chaos Solitons Fractals 35 (2008) 1009-1014. 